\title{
BMJ Open Investigation of the relation between risk assessment of exposure and nosocomial SARS-CoV-2 transmission in healthcare workers: a prospective single-centre study
}

\author{
Ayse Kaya Kalem (D , , Bircan Kayaaslan, ${ }^{1}$ Fatma Eser, ${ }^{1}$ İmran Hasanoglu, ${ }^{1}$ \\ Muge Ayhan, ${ }^{2}$ Belgin Coskun, ${ }^{2}$ Rahmet Guner ${ }^{1}$
}

To cite: Kaya Kalem A, Kayaaslan B, Eser F, et al. Investigation of the relation between risk assessment of exposure and nosocomial SARS-CoV-2 transmission in healthcare workers: a prospective singlecentre study. BMJ Open 2022;12:e056858. doi:10.1136/ bmjopen-2021-056858

- Prepublication history for this paper is available online To view these files, please visit the journal online (http://dx.doi org/10.1136/bmjopen-2021056858).

Received 01 September 2021 Accepted 04 January 2022

\section{Check for updates}

(C) Author(s) (or their employer(s)) 2022. Re-use permitted under CC BY-NC. No commercial re-use. See rights and permissions. Published by BMJ.

${ }^{1}$ Infectious Diseases and Clinical Microbiology Clinic, Ankara Yıldırım Beyazıt University, Ankara City Hospital, Ankara, Turkey

${ }^{2}$ Infectious Diseases and Clinical Microbiology Clinic, Ankara City Hospital, Ankara, Turkey

Correspondence to

Dr Ayse Kaya Kalem;

dr.aysekaya09@hotmail.com

\section{ABSTRACT}

Objectives Healthcare workers (HCWs) are among the risk groups for COVID-19. Determining transmission routes and risk levels during healthcare is of great importance in preventing nosocomial outbreaks. This study aimed to investigate the frequency of nosocomial transmission and factors affecting the transmission in HCW.

Methods HCWs admitted to the infectious diseases outpatient clinic due to contact with a COVID-19 patient and diagnosed with SARS-COV-2 by reverse-transcriptase PCR (RT-PCR) between 20 March 2020 and 30 June 2020 were included in the study.

Results A total of 822 HCWs with 295 low, 284 intermediate and 243 high-risk exposures were included in the study. $27.1 \%$ of the HCWs were male, and the median age was 31.9 years (20-62). $89.5 \%$ of these patients were directly in charge of patient care. Of the index cases contacted, $72.6 \%$ were HCW, and $27.4 \%$ were non-HCW patients. Most of the risky exposure $(51.7 \%)$ occurred in nurses. The occurrence frequency of high-risk exposure was lower in those assigned to direct patient care when compared with the occurrence frequency of moderate-risk or low-risk exposures $(76.5 \%, 94.7,95.3$, respectively $\mathrm{p}<0.001)$. In most high-risk exposures (220/253), the index cases were HCWs $(p<0.001)$. Symptoms were detected in 311 of the HCWs (37.8\%) during the follow-up. The median time to perform SARS-CoV-2 RT-PCR was 5.3 days $(\mathrm{IQR})$ after the last risky exposure. In multivariate analysis, SARS-CoV-2 RT-PCR positivity was 5.65 times higher in HCWs not directly involved in patient care than HCWs who are not involved in patient care $(95 \% \mathrm{Cl} 2.437$ to $13.111 ; p<0.001$ ).

Conclusions This study provides particularly useful information on post-exposure COVID-19 follow-up and management of working schedules and procedures of HCWs.

\section{INTRODUCTION}

While the COVID-19 pandemic continues unabated, healthcare workers (HCWs) at the forefront who are in contact with and caring for COVID-19 patients are among the
Strengths and limitations of this study

- This study was planned prospectively.

- This study had a large sample size.

This study explores the hospital-acquired transmissions in healthcare workers (HCWs) by using standardized risks classification.

- Compliance with other infection control measures such as hand hygiene was not investigated in our study.

- It is possible that positivity was not detected in some asymptomatic or mildly symptomatic individuals since not all exposed HCWs have been screened with the SARS-CoV-2 PCR test.

high-risk groups in terms of COVID-19 transmission. ${ }^{1-4}$ Nosocomial transmission remains to cause anxiety in healthcare professionals who struggle with many factors such as excessive working hours, psychological stress, extreme fatigue, occupational burnout and stigma. SARS-CoV-2 infection is known to be transmitted by respiratory droplets. Direct contact and aerosol-generating procedures (AGP) constitute the highest risk in terms of contamination, especially in departments with confirmed or suspected COVID-19 patients.

China reported the number of infected HCWs as 3387, The Italian National Institute of Health as 17000, and the USA as 9200. ${ }^{5-7}$ A review published in December 2020 stated that $3.9 \%(152,888)$ of COVID-19 patients in the world were HCWs. ${ }^{8}$ However, there are still countries that have not yet reported the number of infected healthcare personnel, and studies investigating risky behaviour within HCWs are very limited. In the nosocomial transmission of SARS-CoV-2, adherence of HCWs to infection prevention 
and control measures and appropriate use of personal protective equipment (PPE) are as significant as the virus characteristics.

The protection of HCWs is one of the most critical points in dealing with the pandemic. Therefore, determining the dynamics of nosocomial transmission within the group of HCWs is of great importance in preventing nosocomial outbreaks and protecting HCWs from infection.

This study aimed to investigate the incidence of nosocomial transmission and the factors affecting the transmission in healthcare professionals admitted to the Infection Control Committee (ICC) due to exposure to COVID-19 patients in our hospital.

\section{MATERIAL AND METHOD \\ Study design}

HCWs admitted due to exposure to a definite COVID-19 patient between 20 March 2020 and 30 June 2020 were included in the study. Written consent was not obtained from the participants since only epidemiological surveillance data were collected.

\section{Infection control}

At the beginning of the pandemic, all HCWs working in our hospital were trained by ICC doctors and nurses on COVID-19 transmission, prevention from the infection, appropriate PPE use, infection control measures and hand hygiene. During the pandemic, all necessary PPEs were provided at an adequate level. It was planned for all HCWs to work with surgical masks during the pandemic period and access appropriate PPEs when necessary. Moreover, the course of action to be followed after a risky exposure was determined, and follow-up forms were created to monitor HCWs with occupational exposure.

\section{Determination of contact type and risk level}

HCW risk assessment and follow-up were performed by ICC with active surveillance. The demographic characteristics (age, gender and chronic disease) of the exposedHCWs, their professions (doctor, nurse, auxiliary health personnel, other auxiliary health personnel, support personnel and administrative staff), and whether they were directly involved in patient care were recorded. Furthermore, along with index case detection, the following data related to the exposure were recorded: index case's mask usage during contact, dates of exposure and PPE usage of the HCW during exposure.

Types of exposures listed in box 1 were considered risky for SARS-CoV-2 transmission, and HCWs that had undergone such exposure were followed up prospectively. Other types of exposures were categorised as 'non-risky' and were excluded from follow-up. ${ }^{910}$

WHO guideline was used to determine whether the appropriate PPE was worn. ${ }^{10}$ The risk level was determined according to PPE usage of the exposed HCW (table 1). Except for AGP, the use of a surgical mask was

\section{Box 1 Risky exposed}

A. Close contact

1. Being at a distance of less than $1 \mathrm{~m}$ with COVID-19 patients for 15 min or more in the last 5 days.

2. Meeting face to face with a COVID-19 patient at a distance of less than $1 \mathrm{~m}$ for $15 \mathrm{~min}$ or longer in the last 5 days.

3. Direct contact with a COVID-19 patient (eg, handshake) or direct contact with the person's secretions (eg, coughing or touching used tissue) in the last 5 days.

\section{B. Intense contact}

1. Performing AGP to a COVID-19 patient or assisting in this process.

AGP, aerosol-generating procedure.

considered sufficient. AGP was defined as respiratory tract sampling, intubation, aspiration of respiratory tract secretions, non-invasive mechanical ventilation, high flow oxygen therapy, cardiopulmonary resuscitation, use of nebuliser, endoscopic procedures, bronchoscopy, videolaryngoscopy, dental practices, examinations of the mouth, throat and nose, ophthalmological examinations, and central catheter insertion. ${ }^{1011}$

\section{Follow-up of exposed HCWs}

HCWs included in the study were followed for symptoms for 14 days after the last risky exposure. Symptoms such as fever $\left(\geq 38^{\circ} \mathrm{C}\right)$, shortness of breath, cough, sore throat, nasal congestion or newly-onset loss of smell were considered suspicious symptoms for COVID-19 disease. ${ }^{11}$ Nasopharyngeal swab samples were taken for SARS-CoV-2 reverse-transcriptase PCR (RT-PCR) to diagnose COVID-19 from cases with COVID-19-related symptoms during their follow-up. SARS-CoV-2 RT-PCR was performed on the seventh day after the risky exposure among asymptomatic exposed-HCWs with moderate or high-risk exposure (table 2). A 7-day work restriction was applied to HCWs who had high-risk exposure after intense contact. Those with negative SARS CoV-2 RT-PCR test on the seventh day returned to work, and their 14 days follow-ups were discontinued. Negative SARS-CoV-2 RT-PCR tests of HCWs with persistent symptoms were repeated 48 hours after the initial test.

\section{Definitions}

A HCW was defined as all personnel working in healthcare facilities, regardless of their involvement in direct patient care. However, the personnel were grouped according to whether they were directly involved in patient care or not. Occupational groups were recorded as doctors, nurses, auxiliary health personnel, other auxiliary health personnel, support personnel and administrative staff. The technicians working in radiology, portable $\mathrm{X}$-ray, laboratory, anaesthesia and physical therapy, biologists and physiotherapists were defined as auxiliary HCWs. Other auxiliary health personnel consisted of staff working in the cafeteria, security, waste services, hospital drivers and secretaries. The group defined as support 


\begin{tabular}{|c|c|c|}
\hline Index case mask-wearing status & PPE using status of $\mathrm{HCW}$ & Risk level \\
\hline \multirow[t]{3}{*}{ No } & Did not use a surgical mask or N95 & High \\
\hline & Did not use eye protection & Low \\
\hline & Did not use gloves and aprons & Low \\
\hline \multirow[t]{4}{*}{ Yes } & $\begin{array}{l}\text { Did not use a medical mask or N95 or } \\
\text { Used a surgical mask in case of N95 indication }\end{array}$ & Moderate \\
\hline & Did not use eye protection & Low \\
\hline & Did not use gloves and aprons & Low \\
\hline & Used all PPE properly & No \\
\hline
\end{tabular}

HCW, healthcare worker; PPE, personal protective equipment.

personnel were those who helped nurses and doctors in the patient care, cleaning the patient room and transferring the patient between units. Apart from this, personnel who did not have direct contact with the patient and took part in administrative tasks in a separate unit were classified as administrative staff. Index case describes a case with a positive SARS-CoV-2 RT-PCR result (source of COVID-19 exposure). The index cases were also classified as patients and HCWs.

\section{Patient and public involvement}

No patients were involved.

\section{Analysis}

HCW exposures identified as low, moderate and high risk were compared against demographic characteristics such as age, gender, comorbid diseases, occupation, involvement in direct patient care, the index case, exposure type (ie, risk level). In our study, the SARS-CoV-2 RT-PCR test was not planned to be performed on low-risk asymptomatic HCWs. However, it was determined from the electronic database that some HCWs had SARS-CoV-2 RT-PCR test, and this information was also recorded. Afterward, follow-up and COVID-19 development characteristics were compared according to risk groups. The correlation between the positivity of SARS-CoV-2 RT-PCR and the risk level was compared between the tested health personnel and had negative and positive results. In addition, factors affecting SARS-CoV-2 RT-PCR positivity were investigated.

\section{Statistical analysis}

All statistical analyses were performed using SPSS for Windows V.18 (SPSS). Kolmogorov-Smirnov test was used to assess the normality assumption. The continuous variables that did not have normal distribution were expressed as median (minimum-maximum) values. Categorical variables were summarised as counts (percentages). For nonnormally-distributed continuous variables, differences between groups were tested using the Kruskal-Wallis test. Pearson $\chi^{2} /$ Fisher's exact test determined the relationship between categorical variables. Univariate logistic regression analysis was used to analyse the factors leading to SARS- CoV-2 PCR positivity. The variables considered in the analyses for SARS- CoV-2 PCR positivity were age, sex, profession, involvement in direct patient care, the

Table 2 Exposed-HCW follow-up

\section{Risky exposure type and risk} level

Intense contact-high risk

\section{Management}

1. Symptom follow-up is performed by isolating at home for 7 days.

2. If the symptom develops, the SARS-CoV-2 RT-PCR test is performed on the symptom day, if no symptoms develop, on the seventh day after the last risky exposure.

3. Those with negative test results start to work and are followed up in terms of symptoms for 14 days.

Intense contact-moderate risk

1. Works with a mask on. Active symptom follow-up is performed.

Close contact-high/moderate risk 2. If the symptom develops, the SARS-CoV-2 RT-PCR test is performed on the symptom day, if no symptoms develop, on the seventh day after the last risky exposure.

Close/intense contact-low risk

1. Works with a mask on.

2. If the symptom develops, the SARS-CoV-2 RT-PCR test is performed on the symptom day.

HCW, Healthcare worker; PPE, Personal protective equipment; RT-PCR, reverse-transcriptase PCR. 


\begin{tabular}{|c|c|}
\hline & HCW $n=822(\%)$ \\
\hline Median age (years) min-max & $31.9(20-62)$ \\
\hline Gender (male) & $223(27.1)$ \\
\hline \multicolumn{2}{|l|}{ Profession } \\
\hline Nurse & $425(51.7)$ \\
\hline Doctor & $180(21.8)$ \\
\hline Supportive personnel & $91(11)$ \\
\hline Other auxiliary health personnel & $66(8.1)$ \\
\hline Auxiliary health personnel & $33(4.1)$ \\
\hline Administrative staff & 27 (3.2) \\
\hline Taking part in direct patient care & $736(89.5)$ \\
\hline Underlying disease & $111(13.5)$ \\
\hline \multicolumn{2}{|l|}{ Exposed-index case } \\
\hline $\mathrm{HCW}$ & $597(72.6)$ \\
\hline Patient & $225(27.4)$ \\
\hline \multicolumn{2}{|l|}{ Risky exposed } \\
\hline Close contact & $785(95.5)$ \\
\hline Intense contact & $37(4.5)$ \\
\hline \multicolumn{2}{|l|}{ Risk level } \\
\hline Low risk & 295 (35.9) \\
\hline Moderate risk & $284(34.5)$ \\
\hline High risk & $243(29.6)$ \\
\hline
\end{tabular}

All data are given as a number (percentage) unless specifically stated. Underlying diseases (patients number): chronic respiratory disease (26), chronic cardiovascular disease (23), thyroid disease (17), chronic rheumatological disease (13), allergic diseases (9), diabetes mellitus (6), chronic neurological disease (5), other (12). HCW, Healthcare workers

index case, index case mask usage, HCWs PPE usage, and risk level. A two-sided $\mathrm{p} \leq 0.05$ was considered statistically significant. A multivariable logistic regression model was used to predict potential risk factors of SARS-CoV-2 PCR positivity. The variables with a significance level of $\mathrm{p} \leq 0.20$ from the univariate analysis were identified as candidate variables for the multivariable model.

\section{RESULTS}

During the study period, a total of 1268 HCWs were admitted with suspicion of risky exposure. After the initial evaluation, exposures that had not met the criteria for risky exposure for COVID-19 were excluded from the follow-up. A total of $822 \mathrm{HCW}$ contacts were classified as risky and were followed up prospectively. Two hundred and ninety-five of these exposures were low risk, 284 were intermediate risk and 243 were high risk. The median age was 31.9 years (20-62), and $27.1 \%$ of exposed HCWs were male. Risky exposure was detected most frequently in nurses $(51.7 \%)$. Of the exposed HCWs, $89.5 \%$ were directly involved in patient care. The index cases were HCWs in $72.6 \%$ of risky exposures and COVID-19 patients in $27.4 \%$. Concerning contact types, $95.5 \%$ were identified as close contact and $4.5 \%$ as intense contact. Demographic and clinical characteristics of exposed HCWs are shown in table 3 .

The comparison of the groups by their risk levels is summarised in table 4 . There was a statistically significant difference between age, comorbid diseases, occupation, direct involvement in patient care, the index case, and risky exposure type $(\mathrm{p}<0.001, \mathrm{p}=0.011, \mathrm{p}<0.001, \mathrm{p}<0.001$, $\mathrm{p}<0.001, \mathrm{p}<0.001$, respectively). As a result of the dual comparison of the groups, the median age was higher in HCWs with high-risk exposure than in the low-risk and moderate-risk group, and the comorbidity rate was higher than in low-risk level $(\mathrm{p}<0.001, \mathrm{p}=0.011$, respectively). As a result of the dual comparison of the groups, the median age was determined to be higher in HCWs with high-risk exposure than in the low-risk and moderate-risk group $(\mathrm{p}<0.001)$. The comorbidity rate was higher in HCWs with high-risk exposure than in the low-risk group $(\mathrm{p}=0.011)$. High-risk exposure was lowest in nurses $(21.9 \%)$ and highest in other assistant healthcare personnel (77.3\%) $(\mathrm{p}<0.001)$. High-risk exposure was lower in those directly involved in patient care $(76.5 \%, \mathrm{p}<0.001)$. In the vast majority of high-risk exposures (220/253), the index case was an HCW.

Post-exposure follow-up features by risk levels are shown in table 5. Symptoms developed during follow-up in a total of $311(37.8 \%)$ HCWs. The most common symptoms were sore throat $(24.2 \%)$ and cough $(14.5 \%)$. A higher rate of complaints occurred in the moderate and high-risk group than in the low-risk exposed group $(\mathrm{p}=0.001)$. During the study, the SARS-CoV-2 RT-PCR test was performed on $59.7 \%(176 / 295)$ of low-risk HCWs, $74.6 \%(212 / 284)$ of medium-risk HCWs and $74.9 \%$ of high-risk HCWs (182/243). SARS-CoV-2 PCR positivity was detected in 28 exposed HCWs. The rate of SARSCoV-2 RT-PCR positivity development after high-risk exposure was higher than both moderate-risk and low-risk exposure groups $(7.1 \%, 3.3 \%, 4.5 \%$, respectively), but the difference was not found to be statistically significant $(\mathrm{p}=0.205)$. SARS-CoV-2 RT-PCR was tested in $58.9 \%$ (301) of 511 asymptomatic HCWs and 86.4\% (269) of 311 symptomatic HCWs. SARS-CoV-2 RT-PCR positivity rates were $3.3 \%(10 / 301)$ and $6.7 \%(18 / 269)$ between asymptomatic and symptomatic HCW, respectively. No statistically significant difference was determined between SARSCoV-2 RT-PCR positivity and risk levels in asymptomatic and symptomatic HCWs (table 5).

When exposed HCWs with and without SARS-CoV-2 RT-PCR positivity were compared; age, gender, index case type, index case mask usage, HCW's PPE usage and contact type were not found as independent risk factors for the development of PCR positivity. The risk of developing COVID-19 was observed to be 5.65 times higher in those who were not directly involved in patient care (OR 5.65, 95\% CI 2.437 - 13.11; $\mathrm{p}<0.001)$. 
Table 4 Comparison of exposed-HCWs by the risk leve

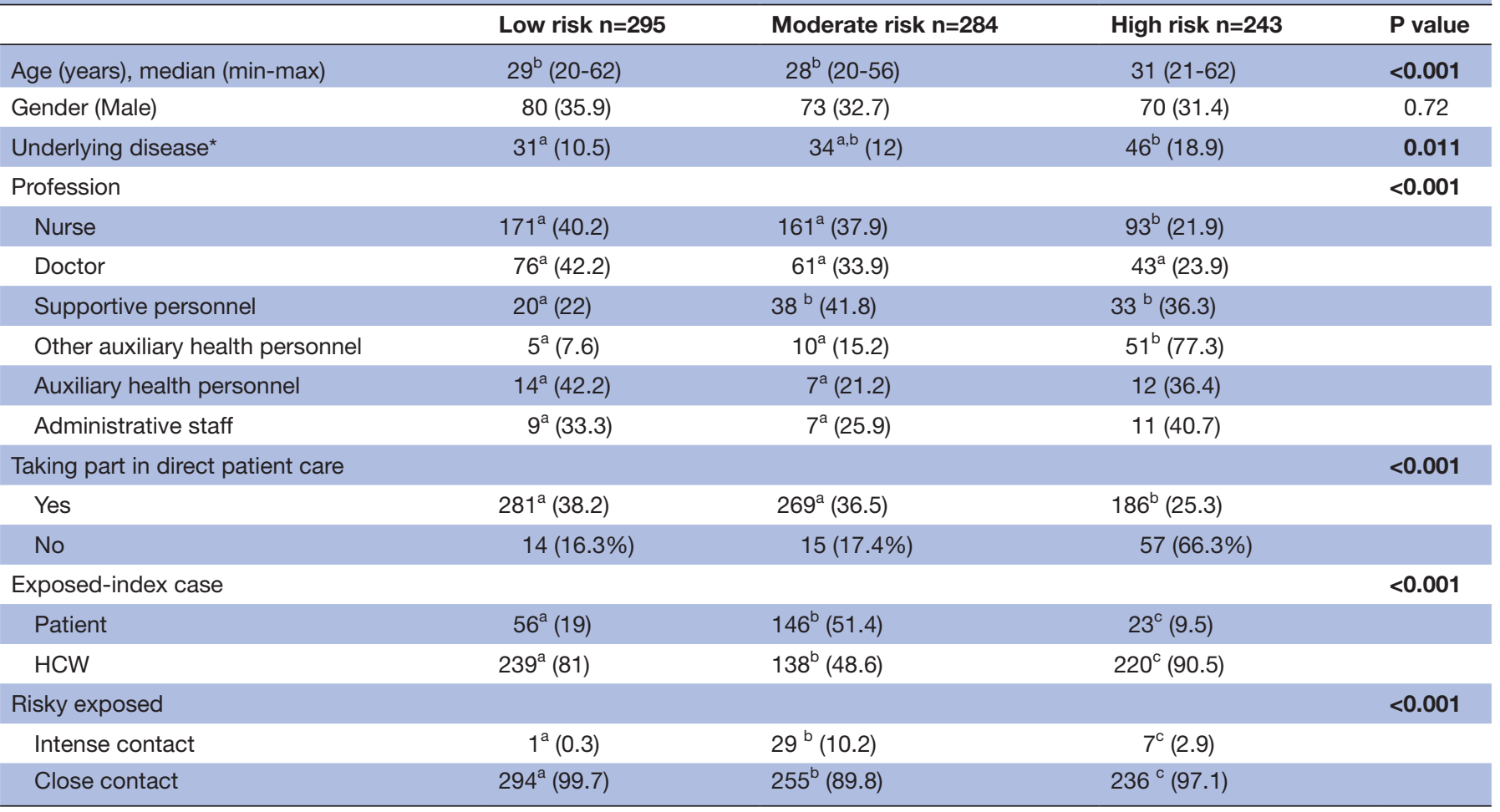

All data are given as a number (percentage) unless specifically stated.

$\mathrm{a}, \mathrm{b}, \mathrm{c}$ There is a difference between the groups indicated by different indices.

Bold values indicate statistical significance.

$\mathrm{HCW}$, healthcare worker.

\section{DISCUSSION}

Protection HCWs who are at high risk due to COVID-19 is vital in fighting the pandemic. In the face of a new disease, the anxiety and fear of HCWs have decreased with the elimination of uncertainty, the increase in knowledge about the means of transmission and prevention measures and the acceleration of vaccination studies. However, during this period, many HCWs were infected

Table 5 Postcontact follow-up results by the risk level

\begin{tabular}{|c|c|c|c|c|}
\hline & Low risk $n=295(\%)$ & Moderate risk $n=284(\%)$ & High risk $n=243(\%)$ & $P$ value \\
\hline Throat ache & $51^{a}(17.3)$ & $79^{\mathrm{b}}(27.8)$ & $69^{b}(28.4)$ & 0.002 \\
\hline Diarrhoea & $8^{a}(2.7)$ & $8^{a}(2.8)$ & $18^{\mathrm{b}}(7.4)$ & 0.009 \\
\hline Fever & $5^{a}(1.7)$ & $8^{\mathrm{a}}(2.8)$ & $19^{\mathrm{b}}(7.8)$ & 0.001 \\
\hline Inability to taste/smell & $1(0.3)$ & $1(0.4)$ & $2(0.8)$ & 0.089 \\
\hline $\begin{array}{l}\text { Duration to test after last risky exposure, } \\
\text { days, median (min-max) }\end{array}$ & $5(1-12)$ & $6(1-18)$ & $5(1-14)$ & 0.065 \\
\hline SARS-CoV-2 RT-PCR positivity & $8(4.5)$ & $7(3.3)$ & $13(7.1)$ & 0.205 \\
\hline In asymptomatic HCW & $4(3.7)$ & $1(0.9)$ & $5(6)$ & 0.191 \\
\hline
\end{tabular}

All data are given as a number (percentage) unless specifically stated.

$a, b, c$ There is a difference between the groups indicated by different indices.

Bold values indicate statistical significance.

$\mathrm{HCW}$, healthcare worker; RT-PCR, reverse transcriptase-polymerase chain reaction. 
with SARS-CoV-2, and a considerable number of them died. Knowledge on COVID-19 infection rates in HCWs and epidemiological dynamics of the infection is still insufficient. The knowledge, skills and adaptation of healthcare professionals regarding infection control measures and PPE use vary widely among HCWs. The concerns that COVID-19 vaccines do not eliminate the infection development, the infections developing with mutant strains, and the decrease in vaccine protection against these mutant strains remind once again the importance of dealing with the characteristics of the infection development in HCWs in more detail. In this study, which was carried out in HCWs applied to ICC due to risky exposure with COVID-19 patients, we aimed to obtain more detailed epidemiological data regarding behaviours carrying risk for HCWs, to evaluate the exposure risks in detail and nosocomial SARS-CoV-2 transmission.

Considering that the increase in knowledge and experience with age and comorbid chronic diseases are risk factors for the poor prognosis of COVID-19, HCWs of older ages and with chronic diseases are expected to be more prudent on measures to prevent infection transmission and appropriate PPE use. However, in our study, the ages of those who have undergone high-risk exposures were higher than the HCWs with low-risk and moderate-risk exposure $(\mathrm{p}<0.001)$ and had a higher rate of comorbid chronic diseases $(\mathrm{p}=0.011)$. Similarly, in the study of Maltezou et al, chronic diseases were more common in high-risk exposures when compared with lowrisk exposures $(2.5 \%-1.5 \%, \mathrm{p}=0.001) .{ }^{12}$

A vast majority of HCWs admitted to ICC with a risky exposure were directly involved in patient care, and most of them were nurses and doctors. This situation can be explained by the fact that nurses are more involved in patient care than other occupational groups and the number of nurses working in the health institution is higher than other personnel. However, in terms of risk levels, it was observed that the rate of high-risk exposures was less in doctors $(23.9 \%)$ and nurses $(21.9 \%)$. In the study of Maltezou et al, unlike our results, it was reported that high-risk exposure was more common in nurses and doctors $(43.4 \%$ vs $36.1, \mathrm{p}<0.001)$. Similar to our study, it was found that high-risk exposure was higher among administrative staff in the same study. ${ }^{12}$ In our country, after the first case was reported in March 2020, it has become obligatory for all hospital personnel to work with a mask. Adherence to this measure reduces many exposures to moderate and low-risk categories. However, the occurrence of high-risk exposure among administrative staff, auxiliary HCWs and other auxiliary HCWs suggests that they suffer from the misconception that they are under less risk in terms of infection development due to not directly taking part in patient care and that often do not adhere to use of masks. A possible reason for this is lower awareness of the fact that infections can be transmitted from colleagues. It seems that the appropriate use of PPE is lower in these groups, and proper training and information sharing should be made individually and more emphatically. Although it can be thought that a high percentage of moderate and high-risk exposure in the support personnel is due to their tasks directly involving patient care, the fact that the index cases are mostly HCWs rather than the patients does not support this idea.

One of the striking findings of the study is the index cases. In $72.6 \%$ of contacts, the index cases were HCWs. Similar to our results, previous studies have shown that HCWs had mostly risky exposure with their colleagues, and most of their exposures developed during eating and drinking activities. ${ }^{12} 13$ The fact that lowest rates of highrisk exposure were observed in contacts with patients and among HCWs directly involved in patient care indicate that HCW's are better in adhering to PPE use in contact with COVID-19 patients. However, they do not pay enough attention to PPE use and infection control measures in their contacts with colleagues.

The SARS-CoV-2 RT-PCR status became positive in the median 5 days after risky exposure, consistent with the disease incubation period. In the study of Maltezou et al, the infection had developed at the end of the first week after risky exposure, and the authors stated that a 7-day work restriction is sufficient. ${ }^{12}$ The WHO and the Centers for Disease Control and Prevention recommend a 14-day work restriction regardless of the risk level. ${ }^{19}$ In our study, in line with COVID-19 guidelines of the Ministry of Health, only 7 days of work restriction was applied after intense high-risk exposure ${ }^{11}$ and only seven HCWs with high-risk and intense exposure underwent a work restriction. Other personnel continued to work with masks, and a super-spreader was not detected. When the need for HCWs has increased, countries must form policies regarding the protection of HCWs based on their internal dynamics.

Although at least one symptom developed in $37.8 \%$ of the risky occupational exposures during the study, SARSCoV-2 PCR positivity was detected in much less of the cases. In the study by Maltezou $\mathrm{et} \mathrm{al}$, at least one symptom was detected in $22.2 \%$ of 3398 HCWs exposed to SARSCoV-2. Symptom development was lower in HCWs with low-risk exposure than in the other two groups with moderate and high risk. Unsurprisingly, the positivity of SARS-CoV-2 after high-risk exposure was higher, but there was no statistically significant difference between the groups. The study of Maltezou et al indicated that the development of COVID-19 after high-risk exposure was six times higher than moderate and low-risk exposures $(5 \%$ in high risk, and $1 \%$ in moderate and low risk, $\mathrm{p}<0.001) .{ }^{12}$ Infection development is expected to be higher in highrisk exposure. The lack of difference between the groups in terms of infection development suggests the transmission may occur through direct exposure due to not paying enough attention to hand hygiene despite the use of appropriate equipment. Moreover, the fact that all HCWs participating in the study were not tested may have affected these rates.

Among the factors evaluated for the development of SARS-CoV-2 positivity, only providing direct care 
to COVID-19 patients was statistically significant, and contrary to expectations, infection development was found 5.65 times higher in HCWs who did not directly provide care for COVID-19 patients. This finding suggests that the staff caring for a COVID-19 patient adhere more strictly to PPE and other infection control measures, while the staff who do not provide direct care do not comply with the measures adequately with a false sense of safety. In addition, 28 HCWs with positive SARS-CoV-2 RT-PCR tests were included in the comparison. The low number of participants should be considered when interpreting our results. In the study of Hunter et al, symptomatic HCWs were divided into three groups: HCWs involved in direct patient care (group 1), HCWs not directly involved in patient care that work in high-risk areas such as laboratories (group 2), and non-clinical workers (group 3). SARS-CoV-2 positivity was detected at a rate of $15 \%(128$ of 834 ) in group 1, $16 \%$ (14 of 86 ) in group 2 and $18 \%$ (20 of 109) in group 3. Furthermore, taking part in direct patient care was not found to be risky for SARS-CoV-2 RT-PCR positivity (group 1 vs group 2: OR 1.08, 95\% CI 0.59 to 1.97 ; group 1 vs group 3: $1.24,95 \%$ CI 0.74 to 2.09; $\mathrm{p}=0.71) .{ }^{14}$ The researchers have drawn attention to community transmission since the study was conducted before the restrictions in society. In studies from Spain and England, no difference was found between the administrative staff and the personnel working in direct patient care in terms of infection development, and it was stated that in-house or community transmission was more effective in HCW infections. ${ }^{14}{ }^{15}$ In a study from France, the infection rate was significantly higher in HCWs who did not directly provide care for COVID-19 patients $(\mathrm{OR}=2.3, \mathrm{p}=0.005) .{ }^{16}$ Similarly, in a study conducted in Germany, the fact that only $3 \%$ of $86 \mathrm{HCWs}$ with a positive SARS-CoV-2 RT-PCR test had contact with COVID-19 patients was accepted as a supporting finding for community transmission. ${ }^{17}$ On the contrary, in a study conducted in Wuhan, 72 exposed HCWs were examined, and it was found that HCWs working in areas with COVID-19 patients were 2.13 times more under risk. ${ }^{18}$ However, in this study, the number of personnel exposed to SARSCoV-2 is very low. Besides, since it is the emerging point of the pandemic, the dynamics of HCWs' adherence to the infection control measures in contact with patients and each other may differ compared with other studies.

In addition to the use of PPE, the adherence of HCWs to other infection control measures such as hand hygiene and unknown/possible community transmission may have contributed to the difference between the groups. Studies related to HCW risk factors indicate that practicing suboptimal hand hygiene before and after patient exposure, long working hours, inappropriate PPE use and PPE insufficiency, inadequate training on infection control measures, and the unit where an HCW is employed were found to be risk factors for COVID-19 transmission in HCWs. Previous studies report failure to evaluate the effect of remembering bias and other environmental factors as limitations. ${ }^{13}$ 18-27 This study was planned prospectively, and negativities such as possible false recall and lack of data, which are among the weaknesses of retrospective studies, were minimised. Also, more categorised information has been obtained by using a standardised risk classification in the follow-up and management of exposed HCWs. However, there are several limitations: Although most exposed HCWs have been screened with PCR, it is possible that positivity was not detected (underestimation) in some asymptomatic or mildly symptomatic individuals since not all exposed HCWs have been screened with SARS-CoV-2 PCR test. Weekly screening of all health personnel is also included in the recommendations. It would be more beneficial to perform these to increase our study's strength. However, it does not seem possible in terms of both cost and laboratory capacity for our hospital, where 15000 SP works and shouldering the pandemic burden of the region. Screenings were performed according to risk level and symptom presence within the scope of national guideline recommendations. Moreover, although exposed-HCWs were followed up prospectively, the risk groups in the study were determined based on HCW's own statements. Another limitation is that compliance to proper PPE usage procedures and adherence to hand hygiene have not been investigated.

\section{CONCLUSIONS}

In this study, many HCWs were actively followed according to a prospective, post-exposure standardised risk classification. HCWs have a high risk of being infected while providing care for COVID-19 patients. However, prevention of the infections that will develop during the contact of HCWs with other hospital employees seems to be a priority. Increasing infection rates among HCWs may lead to health system collapse and worsening of the pandemic. This study provides beneficial information by utilising standardised risk classification of nosocomial transmissions in HCWs. It also provides particularly useful information on postexposure follow-up and required working restrictions for HCWs. The study results revealed that adherence to infection control rules is of vital importance in terms of raising awareness about adherence to PPE usage rules and preventing transmission between personnel. In such a period where the need for HCWs has increased, it will contribute to reorganising regulatory actions by revealing situations carrying the risk of infection.

Collaborators Study group Zehra Acamur Iffet Azapay Cinarbas Burcu Baltacıoglu Emel Bas Cicek Hatice CelikYeliz Derge Halit Dogan Fatma Donmez Bengi Dulek Yasemin Dursun Guler Belgin Erdogan Betul Karahan Kurt Yıldız Kilıc Gulay KocaozGulderen Saylan Ulku Tosun Gonul Ulutas Ayten Un Semra Yesil.

Contributors Conception and design of study: AKK and RG. Acquisition of data: AKK, IH, FE and MA, BC. Analysis and/or interpretation of data: AKK, BK and $\dot{I} H$. Drafting the manuscript: AKK and BK. All authors discussed the results and contributed to the final manuscript. Guarantor: AKK

Funding The authors have not declared a specific grant for this research from any funding agency in the public, commercial or not-for-profit sectors. 
Competing interests None declared.

Patient and public involvement Patients and/or the public were involved in the design, or conduct, or reporting, or dissemination plans of this research. Refer to the Methods section for further details.

Patient consent for publication Not applicable.

Ethics approval This study was approved by Turkish Ministry of Health and Ankara City Hospital Ethical Committee, refference number: E1-20-559.

Provenance and peer review Not commissioned; externally peer reviewed.

Data availability statement Data are available on reasonable request. All data relevant to the study are included in the article or uploaded as online supplemental information. The identified data associated with this study is stored by Ankara City Hospital İnfectious Diseases and Clinical Microbiology department.

Open access This is an open access article distributed in accordance with the Creative Commons Attribution Non Commercial (CC BY-NC 4.0) license, which permits others to distribute, remix, adapt, build upon this work non-commercially, and license their derivative works on different terms, provided the original work is properly cited, appropriate credit is given, any changes made indicated, and the use is non-commercial. See: http://creativecommons.org/licenses/by-nc/4.0/.

\section{ORCID iD}

Ayse Kaya Kalem http://orcid.org/0000-0002-4759-0066

\section{REFERENCES}

1 World Health Organization. Risk assessment and management of exposure of health care workers in the context of COVID-19. Interim guidance, 2020. Available: https://apps.who.int/iris/bitstream/handle/ 10665/331496/WHO-2019-nCov- HCW_risk_assessment-2020.2eng.pdf [Accessed 27 May 2020].

2 Wu Z, McGoogan JM. Characteristics of and Important Lessons From the Coronavirus Disease 2019 (COVID-19) Outbreak in China: Summary of a Report of 72314 Cases From the Chinese Center for Disease Control and Prevention. JAMA 2020;323.

3 Belingheri M, Paladino ME, Riva MA. COVID-19: health prevention and control in non-healthcare settings. Occup Med 2020;70:82-3.

4 Koh D. Occupational risks for COVID-19 infection. Occup Med 2020;70:3-5.

5 Zhan M, Qin Y, Xue X, et al. Death from Covid-19 of 23 Health Care Workers in China [published online ahead of print, 2020 Apr 15]. N Engl J Med 2020;NEJMc2005696.

6 Instituto Superiore di Sanit. Integrated surveillance of COVID-19 in Italy, 2020. Available: https://www.epicentro.iss.it/en/coronavirus/ bollettino/Infografica_17aprile\%20ENG.pdf [Accessed 28 April 2020].

7 CDC COVID-19 Response Team. Characteristics of Health Care Personnel with COVID-19 - United States, February 12-April 9, 2020. MMWR Morb Mortal Wkly Rep 2020;69:477-81.

8 Bandyopadhyay S, Baticulon RE, Kadhum M, et al. Infection and mortality of healthcare workers worldwide from COVID-19: a systematic review. BMJ Glob Health 2020;5:e003097.

9 Centers for Disease Control and Prevention. Interim U.S. guidance for risk assessment and work restrictions for healthcare personnel with potential exposure to COVID-19, 2020. Available: https://www. cdc.gov/coronavirus/2019-ncov/hcp/guidance-risk-assesment-hcp. html [Accessed 27 May 2020].
10 World Health Organization. Health workers exposure risk assessment and management in the context of COVID-19 virus: interim guidance, 4 March 2020. World Health organization, 2020. Available: https:// apps.who.int/iris/handle/10665/331340

11 Halk Sağlığı Genel Müdürlüğü. COVID-19 (SARS-CoV-2 Enfeksiyonu) Rehberi (Bilim Kurulu Çalıșması), 2020. Available: file://C:/Users/10005527/Desktop/Personel\%20COVID-19\%20 takip/COVID19-TemasiOlanSaglikCalisanlarininDegerlendirilmesi05042020.pdf

12 Maltezou HC, Dedoukou X, Tseroni M. Roll-Out of SARS-CoV-2 testing for healthcare workers at a large NHS Foundation trust in the United Kingdom, March 2020. Euro Surveill 2020;25:2000433.

13 Contejean A, Leporrier J, Canouï E, et al. Transmission routes of severe acute respiratory syndrome coronavirus 2 among healthcare workers of a French university hospital in Paris, France. Open Forum Infect Dis 2021;8:ofab054.

14 Hunter E, Price DA, Murphy E, et al. First experience of COVID-19 screening of health-care workers in England. Lancet 2020;395:e77-8.

15 Folgueira MD, Muñoz-Ruipérez C, Alonso-López MA. SARS-CoV-2 infection in health care workers in a large public hospital in Madrid, Spain, during March 2020. MedRxiv 2020.

16 Gagneux-Brunon A, Pelissier C, Gagnaire J, et al. SARS-CoV-2 infection: advocacy for training and social distancing in healthcare settings. J Hosp Infect 2020;106:610-2.

17 Kluytmans M, Buiting A, Pas S. SARS-CoV-2 infection in 86 healthcare workers in two Dutch hospitals in March 2020. medRxiv 2020;3.

18 Ran L, Chen X, Wang Y, et al. Risk factors of healthcare workers with coronavirus disease 2019: a retrospective cohort study in a designated hospital of Wuhan in China. Clin Infect Dis 2020;71:2218-21.

19 Calò F, Russo A, Camaioni C, et al. Burden, risk assessment, surveillance and management of SARS-CoV-2 infection in health workers: a scoping review. Infect Dis Poverty 2020;9:139.

20 Chou R, Dana T, Buckley DI, et al. Update alert: epidemiology of and risk factors for coronavirus infection in health care workers. Ann Intern Med 2020;173:W46-7.

21 Heinzerling A, Stuckey MJ, Scheuer T, et al. Transmission of COVID-19 to Health Care Personnel During Exposures to a Hospitalized Patient - Solano County, California, February 2020. MMWR Morb Mortal Wkly Rep 2020;69:472-6.

22 Celebi G, Pișkin N, Çelik Bekleviç A, et al. Specific risk factors for SARS-CoV-2 transmission among health care workers in a university hospital. Am J Infect Control 2020;48:1225-30.

23 Wang J, Zhou M, Liu F. Reasons for healthcare workers becoming infected with novel coronavirus disease 2019 (COVID-19) in China. J Hosp Infect 2020;105:100-1.

24 Belingheri M, Paladino ME, Riva MA. Beyond the assistance: additional exposure situations to COVID-19 for healthcare workers. $J$ Hosp Infect 2020;105:353.

25 Lentz RJ, Colt H, Chen H, et al. Assessing coronavirus disease 2019 (COVID-19) transmission to healthcare personnel: the global ACTHCP case-control study. Infect Control Hosp Epidemiol 2021;42:1-7.

26 Eyre DW, Lumley SF, O'Donnell D, et al. Differential occupational risks to healthcare workers from SARS-CoV-2 observed during a prospective observational study. Elife 2020;9:e60675.

27 Jin $\mathrm{Y}-\mathrm{H}$, Huang Q, Wang Y-Y, et al. Perceived infection transmission routes, infection control practices, psychosocial changes, and management of COVID-19 infected healthcare workers in a tertiary acute care hospital in Wuhan: a cross-sectional survey. Mil Med Res 2020;7:24. 